\title{
Accessory Embryonic Residual Vein Circulation in Adult Detected during Patent Foramen Ovale Percutaneous Closure
}

\author{
Andrea Annoni ${ }^{1}$, Daniele Andreini ${ }^{1}$ and Daniela Trabattoni ${ }^{1 *}$ \\ ${ }^{1}$ Cardiovascular CT Unit, Centro Cardiologico "Monzino", IRCCS, Institute of Cardiology, University of Milan, Milan, Italy \\ ${ }^{2}$ Department of Invasive Cardiology, IRCCS, Institute of Cardiology, University of Milan, Milan, Italy
}

“Corresponding author: Daniela Trabattoni, Centro Cardiologico "Monzino”, IRCCS, Institute of Cardiology, Via Parea, 4, 20138 Milan, Italy, Tel: +39 02 58002331; Fax: +39 02 58002398; E-mail: daniela.trabattoni@ccfm.it

Rec date: Jul 21, 2014; Acc date: Aug 23, 2014; Pub date: Aug 25, 2014

Copyright: (C) 2014 Annoni A, et al. This is an open-access article distributed under the terms of the Creative Commons Attribution License, which permits unrestricted use, distribution, and reproduction in any medium, provided the original author and source are credited.

\begin{abstract}
Inferior vena cava (IVC) anomalies may display with different anatomical features due to the complexity of the embryogenetic process. These vascular anatomical variants occur in $0.4-3.5 \%$ of patients. We present the case of a 41 year old woman with a previous transient ischemic attack, positive brain Computed Tomography (CT) scan and Magnetic Resonance (MRI) showing an ischemic frontal area and Patent Foramen Ovale (PFO) with right to left shunt. During the advancement of a .035" J-tipped guide wire via the right femoral vein for PFO closure, a paravertebral supplementary venous circulation was detected and confirmed by CT scan. The incidental diagnosis of venous accessory circulation associated with PFO raised the awareness to the existence of these rare anatomical variations for both the clinician and the interventionalist.
\end{abstract}

Keywords: Patent foramen ovale; Percutaneous closure; Supplementary paravertebral circulation

\section{Introduction}

Abnormalities of IVC are relatively common and usually asymptomatic. The complexity of the ontogenesis of the IVC, with numerous anastomoses formed between the primitive paired veins, can lead to a wide array of anomalies in the basic plan of venous return from the lower extremities and abdomen sometimes associated with other cardiac congenital defects $[1,2]$.

Here we present an incidental angiographic detection of a residual embryonic complete venous circulation additional to a fully developed IVC in a patient with a patent foramen ovale.

\section{Case report}

A 41 year old woman with a previous transient ischemic attack occurred with aphasia and paresthesias underwent neurological evaluation with brain CT scan and MRI positive for an ischemic frontal area. Transthoracic echocardiogram showed Patent Foramen Ovale (PFO) with a right to left shunt at baseline and paradoxical shunting during Valsalva maneuver. Contrast transcranial Doppler assessed $>50$ microembolic signals during Valsalva. According to these results, percutaneous closure of PFO was planned. PFO closure was performed by standard technique by right and left femoral vein approach with $8 \mathrm{~F}$ sheath introducers using intraprocedural Intracardiac Echo (ICE) guidance, (9F, $9 \mathrm{MHz}$ Ultra ICE; EP Technologies, Boston Scientific Corporation, San Jose, CA, USA). The $8 \mathrm{~F}$ left femoral vein sheath was exchanged with a $8.5 \mathrm{~F}, 63 \mathrm{~cm}$ long, Slow-Curve introducer (St. Jude Medical, Inc, St. Paul, MN, USA). The Ultra ICE catheter was then advanced into the right atrium allowing for a clear evidence of a bulging septum primum with PFO. No difficulties were observed in catheter advancing along the inferior vena cava while a .035 " J-tipped guide wire inserted via the right femoral and iliac vein stopped at the renal level and contrast injection documented a paravertebral supplementary venous circulation with normal right femoral and iliac veins. Angiography showed an alternative venous pathway originating from distal right iliac vein (Figure 1).

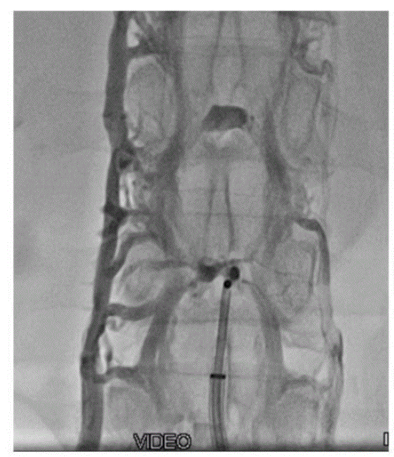

Figure 1: Angiographic findings. Selective venous angiogram performed during PFO closure showing a paravertebral supplementary venous circulation with normal right femoral and iliac veins.

Therefore, a .035" J-tipped guide wire was retracted from the anomalous vein and advanced via the right femoral vein through the PFO into the upper pulmonary vein. An Amplatzer PFO occluder (AGA Medical Corporation, Golden Valley, MN) $25 \mathrm{~mm}$ in size was selected according to the atrial septum and fossa ovalis characteristics and deployed under fluoroscopy and ICE guidance. ICE was performed after device deployment to check for adequate positioning of the device, possible obstruction to systemic or pulmonary venous return and impairment of the atrioventricular valves. The device was 
then released by counterclockwise rotation of the delivery cable. A control transthoracic echo was performed 24 hours later assessing the correct device position and no residual shunt. A total body CT scan showed a paravertebral venous circulation with two vessels sizing 7 $\mathrm{mm}$ and draining into the azygos vein (Figure 2). These accessory vessels seem to derive from persistance of paired embryonic posterior cardinal and subcardinal veins.
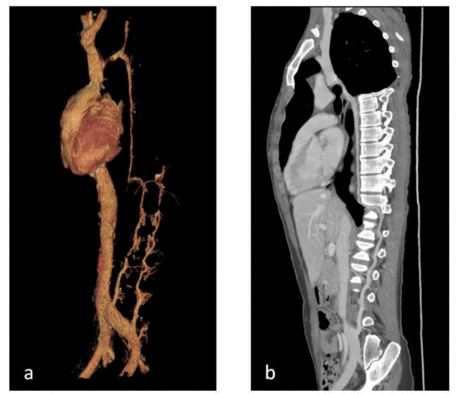

Figure 2: CT Findings. a. CT Volume rendering image of the persistent posterior cardinal and subcardinal veins with paravertebral circulation, b. CT curved multi planar reconstruction image showing accessory venous vessel course from the iliac vein to the azygos vein

\section{Discussion and Conclusion}

Azygos vein as continuation of the IVC results from a failure to form the right subcardinal hepatic anastomosis with atrophy at the right subcardinal vein. Blood is shunted from the suprasubcardinal anastomosis through the retrocrural azygos vein $[1,2]$. IVC anomalies have a propensity to cause lower limb deep vein thrombosis (DVT) [3]. Although rarely suspected, recent studies have revealed that IVC anomalies are not so rare and display with different anatomical features due to the complexity of the embryogenesis of IVC. Moreover, a contrast-enhanced CT scan or an MRI is the only specific method to identify vascular anomalies, which are present in $0.4-3.5 \%$ of patients, given that nearly all of these patients are clinically asymptomatic. Unknowing these vascular abnormalities could lead to high risk of recurrent pulmonary embolism despite IVC filter positioning, to severe haemorrhages during surgical interventions on the retroperitoneal, cardiac and aesophageal regions [4]. In our case, incidental detection of venous accessory circulation associated with patent foramen ovale raised the awareness to the existence of these rare anatomical variations for both the clinician and the interventionalist.

\section{References}

1. Phillips E (1969) Embryology, normal anatomy, and anomalies, Venography of the inferior vena cava and its branches. Williams \& Wilkins, Baltimore.

2. Giordano JM, Trout HH 3rd (1986) Anomalies of the inferior vena cava. J Vasc Surg 3: 924-928.

3. MILLOY FJ, ANSON BJ, CAULDWELL EW (1962) Variations in the inferior caval veins and in their renal and lumbar communications. See comment in PubMed Commons below Surg Gynecol Obstet 115: 131-142.

4. Kumar S (2006) An anomaly of inferior vena cava: a rare case report. See comment in PubMed Commons below Kathmandu Univ Med J (KUMJ) 4: 253-255. 